\title{
Problemi di corrosione e incrostazione della colonna pozzo.
}

\author{
Nicola Cempini \\ Ingegnerie Toscane srl. \\ n.cempini@ingegnerietoscane.net
}

La colonna pozzo in acciaio, sia al carbonio che inox, può essere sottoposta a fenomeni di corrosione e/o incrostazione dovuti alle caratteristiche dell'acqua, alla presenza di correnti vaganti, all'innescarsi della corrosione galvanica.

Quest'ultima avviene qualora due metalli o parti dello stesso metallo a diverso potenziale, vengano posti in opera in contatto in presenza di un elettrolita quale l'acqua di falda; si forma una cella galvanica in corto circuito: gli atomi di ferro metallico dell'acciaio trovano le condizioni energetiche favorevoli per passare in soluzione nell'acqua come cationi di ferro ferroso. La parte di metallo meno nobile (a potenziale minore) si ossida; per ogni ione di ferro passato in soluzione corrispondono due elettroni in eccesso che migrano verso la parte di metallo più nobile (il catodo) e poi passano ad una delle due specie riducibili presenti in soluzione: $\mathrm{Fe}^{2+}$ e $\mathrm{O}^{2}$. Siccome l'ossigeno è più ossidante del ferro, si riduce formando ioni ossidrili che reagiscono a loro volta col ferro ferroso formando idrossido ferroso $\mathrm{Fe}(\mathrm{OH})_{2}$, precipitato poco solubile. Se nell'acqua è presente anche ossigeno disciolto l'idrossido ferroso si ossida e forma idrossido ferrico $\mathrm{Fe}(\mathrm{OH})_{3}$ che è un prodotto completamente insolubile con una buona adesione al metallo su cui va a depositarsi; questo fatto si traduce nella formazione di una specie di pellicola protettiva che inibisce il proseguimento del processo corrosivo (Chiesa, 2003).

Lacqua di falda intercettata dal pozzo può risultare incrostante, corrosiva o addirittura indurre contemporaneamente sia corrosione che incrostazione. In generale, il fenomeno più spesso innescato dall'acqua di falda è quello dell'incrostazione, soprattutto quando si tratta di pozzi per acqua fredda; può capitare tuttavia di trovare acque sottosature in carbonato di calcio che devono essere considerate corrosive. Il carbonato di calcio è il più comune prodotto dell'incrostazione ma comunque molto frequenti sono anche gli ossidi di ferro e/o manganese, questi ultimi sovente associati ad attività batterica.

La durezza dell'acqua può essere definita come il suo contenuto in sali di calcio e di magnesio. Alla durezza totale contribuiscono in misura minore alluminio, manganese, ferro e zinco. La durezza relativa al solo contenuto dei carbonati di calcio e magnesio è definita durezza temporanea; il contributo dei non carbonati è normalmente una piccola componente della durezza totale e ai fini della determinazione della capacità corrosiva o incrostante dell'acqua può essere trascurata (Schnieders, 2003).

La quantità di sali di calcio che principalmente determina la durezza dell'acqua di falda è un parametro molto importante in quanto è sulla capacità dell'acqua di depositare o sciogliere carbonato di calcio che si basano le valutazioni sul potere incrostante o corrosivo dell'acqua stessa.

Per determinare il carattere corrosivo o incrostante dell'acqua di falda sono utilizzati comunemente due indici numerici: il Langelier Sauration Index (LSI) e il Ryznar Stability Index (RSI). Entrambi questi indici si basano sul calcolo del $\mathrm{pH}$ di saturazione per il carbonato di calcio $\left(\mathrm{pH}_{\mathrm{s}}\right)$ il quale poi viene messo in relazione col $\mathrm{pH}$ effettivo dell'acqua secondo le seguenti relazioni:

$$
\begin{aligned}
& \mathrm{LSI}=\mathrm{pH}-\mathrm{pH}_{\mathrm{s}} \\
& \mathrm{RSI}=2 \mathrm{pH}_{\mathrm{s}}-\mathrm{pH}
\end{aligned}
$$

LSI e RSI si basano entrambi sull'assunto che quando il carbonato di calcio eccede la quantità che può restare in soluzione allora precipita e che quanto più il $\mathrm{pH}$ è basso tanto più l'acqua risulterà aggressiva. Il fatto che la corrosione si manifesti a bassi livelli di $\mathrm{pH}$ è un assunto che va bene in sistemi idraulici principalmente costituiti da materiali ferrosi. In generale quindi:

$$
\begin{aligned}
& \mathrm{pH}_{\mathrm{s}}>\mathrm{pH} \text { acqua aggressiva } \\
& \mathrm{pH}_{\mathrm{s}}=\mathrm{pH} \text { acqua bilanciata } \\
& \mathrm{pH}_{\mathrm{s}}<\mathrm{pH} \text { acqua incrostante }
\end{aligned}
$$

Per il calcolo di questi indici è necessario conoscere la quantità totale di solidi disciolti, detta TDS, il pH, la durezza e la temperatura all'origine oltre che l'alcalinità totale (di solito espressa in termini di contenuto di bicarbonati). E' possibile trovare molti calcolatori online di questi due indici (ad es. http://www.lenntech.com/calculators/ryznar/index/ ryznar.htm e http://www.lenntech.com/calculators/langelier/index/langelier.htm).

La quantità totale di solidi disciolti è un buon indicatore speditivo della qualità dell'acqua. In genere ad un aumento del TDS corrisponde una maggiore probabilità di avere a che fare con problemi di qualità. La quantità totale di solidi disciolti è in rapporto di proporzionalità diretta con la conducibilità elettrica e quindi può essere stimata sulla base delle misure di conducibilità.

Bisogna prestare attenzione al fatto che mentre l'incrostazione avviene in dipendenza dei fattori TDS - $\mathrm{pH}$ - temperatura - alcalinità - durezza, questi influiscono sulla capacità corrosiva dell'acqua in modo indipendente. Per esempio nel caso dell'LSI, acque con bassa alcalinità, morbide ma con $\mathrm{pH}$ molto alto o molto basso possono essere corrosive anche se l'indice non lo predice. Questo a causa del fatto che non c'è sufficiente quantità di carbonato di calcio e alcalinità per la formazione di una incrostazione protettiva. E' bene utilizzare LSI solo quando l'alcalinità è maggiore di $40 \mathrm{mg} / \mathrm{l}$, c'è una sufficiente concentrazione di calcio e il $\mathrm{pH}$ varia tra 6,5 e 9,5. 
Entrambi questi indici, proprio perché si basano sulla saturazione in carbonato di calcio, sono molto più validi per predire l'incrostazione che non la corrosione. Tuttavia l'indice di Ryznar fornisce predizioni migliori.

Secondo Schnieders (2003) un acqua con alcune delle seguenti caratteristiche deve essere considerata potenzialmente corrosiva:

\begin{tabular}{|c|c|c|c|}
\hline LSI $<-0,5$ & $\mathrm{H}_{2} \mathrm{~S}>1 \mathrm{mg} / \mathrm{l}$ & $\mathrm{O}_{2}>2 \mathrm{mg} / \mathrm{l}$ & $\mathrm{RSI}>7$ \\
\hline $\mathrm{CO}_{2}>50 \mathrm{mg} / \mathrm{l}$ & Cloruri $>200 \mathrm{mg} / \mathrm{l}$ & TDS $>1000 \mathrm{mg} / \mathrm{l}$ & \\
\hline
\end{tabular}

Quando due o più dei precedenti agenti corrosivi si manifesta contemporaneamente, l'alta probabilità dell'attacco corrosivo al metallo deve destare preoccupazione e uno dei possibili indirizzi progettuali per quanto riguarda il casing è senzaltro l'acciaio inox di tipo austenitico. L'AISI 304 è un materiale che mostra un'ottima resistenza agli attacchi corrosivi di natura chimica ma in presenza di acque in condizioni anossiche o di alte concentrazioni di cloruri o alogenuri può subire danni corrosivi consistenti in tempi relativamente brevi. Infatti se l'acqua presenta una forte carenza di ossigeno lo strato di ossido di cromo può avere difficoltà a conservarsi; i cloruri e gli alogenuri (soprattutto lo ione $\mathrm{Cl}^{-}$) spezzano il film passivante. In questo caso occorre orientarsi verso altri acciai come il 316 o il 316L che grazie alla presenza del molibdeno assicurano un migliore resistenza. Sicuramente l'alta percentuale di elementi pregiati come $\mathrm{Ni}, \mathrm{Cr}, \mathrm{Ti}, \mathrm{Nb}$, Ta, rende questo tipo di acciaio piuttosto costoso.

Laltro indirizzo progettuale nel caso di acque fortemente aggressive è senzaltro il materiale termoplastico se naturalmente questo soddisfa le esigenze legate alle sollecitazioni meccaniche.

I materiali termoplastici disponibili per i pozzi attualmente in commercio sono il PVC ed il PPHM; entrambi hanno un'ottima resistenza all'aggressività dell'acqua ed inoltre sono molto meno sottoposti dell'acciaio alle incrostazioni sia minerali che batteriche. I materiali termoplastici cono inoltre insensibili all'azione delle correnti vaganti.

Il fenomeno della corrosione da correnti vaganti è responsabile di notevoli danni a carico delle strutture metalliche interrate, in particolare tubi. Il terreno infatti, a causa della sua umidità permanente e dei Sali in esso disciolti costituisce la fase a conduzione ionica di una vera e propria cella galvanica i cui elettrodi sono due punti diversi della struttura metallica interrata, nel caso in cui questa sia percorsa da una corrente elettrica vagante.

Si definisce corrente vagante una corrente dispersa nel terreno da tutti gli impianti elettrici con presa a terra: linee di trazione elettrica con ritorno su rotaia; impianti radiofonici e telefonici, ecc.

Queste correnti incontrando nel loro percorso un materiale metallico vi penetrano a causa della minore resistività di questo rispetto al terreno e quindi ne fuoriescono; sfruttano il metallo interrato come corsia preferenziale nel loro viaggio di ritorno alla sottostazione.

Poiché il metallo è un conduttore di prima classe, il punto di ingresso della corrente è quello a potenziale elettrico minore (catodo) mentre quello di uscita è a potenziale elettrico maggiore (anodo); dato che la natura chimica del metallo attraversato è la stessa subisce la corrosione la zona a potenziale elettrico maggiore. Il polo positivo si trova, nella tubatura interrata, proprio nel punto di uscita della corrente vagante.

Le lesioni da correnti vaganti sono difficilmente contrastabili con sistemi passivi: le condutture importanti (olio, gas) sono protette in modo attivo col sistema della protezione catodica, facendo percorrere la tubazione da una corrente.

Quando si è in presenza di correnti vaganti perciò, il metodo di contrasto più efficace è quello dell'utilizzo di materiali termoplastici per lo meno fino a profondità di sicurezza. Altrimenti si può realizzare un avampozzo di profondità adeguata, completamente cementato in modo da sfruttare quanto più possibile le caratteristiche di materiale dielettrico e impermeabile.

E' importante in fase progettuale capire per quanto possibile con che tipo di acqua si avrà a che fare: la cosa è naturalmente più semplice sei sta progettando un'opera nuova nell'ambito dell'ottimizzazione e/o dell'espansione di un campo pozzi già esistente mentre è più complicata se il target della perforazione è una falda sconosciuta. E' inoltre importante verificare il contesto ambientale con particolare riguardo alla presenza di correnti vaganti. Occorre infine evitare di porre in opera acciai con caratteristiche diverse.

Come accennato, le acque più comuni sono di tipo incrostante. Se l'acqua è dura e la velocità di ingresso nel pozzo si avvicina troppo o eccede $0,03 \mathrm{~m} / \mathrm{s}$, l'acqua tende a degassare perdendo $\mathrm{CO}_{2}$ e precipitando carbonato di calcio.

Le incrostazioni sono relativamente facili da trattare prima che induriscano e diventino troppo dure e più difficili da trattare. Questo non vale solo per le incrostazioni dovute al carbonato di calcio ma anche per quelle dovute agli ossidi di ferro e manganese.

In generale un'acqua deve essere considerata incrostante se:

\begin{tabular}{|c|c|c|}
\hline $\mathrm{LSI}>2$ & $\mathrm{pH}>7,5$ & $\mathrm{Fe}>0,5 \mathrm{mg} / \mathrm{l}$ \\
\hline Durezza $>30^{\circ} \mathrm{F}$ & $\mathrm{Mn}>0,2 \mathrm{mg} / \mathrm{l}$ & $\mathrm{RSI}<7$ \\
\hline
\end{tabular}

Le incrostazioni su tubi e filtri possono essere rimosse sia per via meccanica che chimica o tutte e due. Pistonaggio, jetting tool e hydropuls sono i metodi meccanici più comuni e sono molto efficaci ma devono essere utilizzati da esperti in quanto una erogazione insufficiente dell'energia non sarebbe efficace e al contrario una erogazione eccessiva potrebbe causare brutte sorprese come il lesionamento del pozzo o addirittura il suo franamento. Il metodo chimico si basa sul lavaggio del pozzo con prodotti destinati alla rimozione delle incrostazioni ed alla disinfezione.

\section{BIBLIOGRAFIA}

G. Chiesa (2003). Problemi di corrosione ed incrostazione nei pozzi per acqua, Geo-Graph.

Schnieders John H. (2003). Chemical Cleaning, Disinfection and Decontamination of Water Wells, Johnson Screens. 